\title{
Bistability of (Ga,Mn)As Ferromagnetic Nanostructures Due to the Domain Walls Switching
}

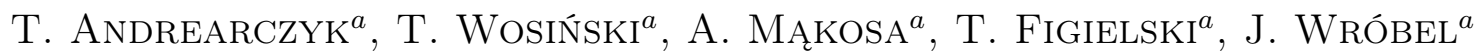 \\ AND J. SADOWSKI ${ }^{a, b}$ \\ ${ }^{a}$ Institute of Physics, Polish Academy of Sciences, al. Lotników 32/46, 02-668 Warsaw, Poland \\ ${ }^{b}$ MAX-Lab, Lund University, 22100 Lund, Sweden
}

\begin{abstract}
We designed and investigated four-arm nanostructures, composed of two perpendicularly crossed stripes, fabricated from ferromagnetic $(\mathrm{Ga}, \mathrm{Mn})$ As layer by means of electron-beam lithography patterning and chemical etching. The nanostructures exhibit a bistable resistance behavior resulting from two configurations of magnetic domain walls in the central part of the structures. We demonstrate a possibility of switching between two stable resistance states in zero magnetic field by applying a pulse of either weak magnetic field or electric current through the structure.
\end{abstract}

PACS numbers: 73.50.Jt, 75.50.Pp, 75.30.Gw, 75.60.Ch, 85.75.-d

\section{Introduction}

Ferromagnetic nanostructures, like nanowires, contain usually a single magnetic domain or a few domains of different magnetization directions separated by domain walls (DWs). A DW contributes an extra electrical resistance to the wire, and its transmission along the wire changes the magnetization direction. The possibility of controlled movement of DWs in ferromagnetic nanowires opens the way for new concepts of spintronics devices, such as magnetic logic gates [1] and nonvolatile memory cells [2]. It is promising for future applications that DWs can be driven not only by a magnetic field, but also by a spin-polarized electric current passing through ferromagnetic wires; see [3] for recent review on the theory of current-driven DW motion. Importantly, in diluted ferromagnetic semiconductors the threshold current for an activation of the DW motion is at least two orders of magnitude smaller than in classical metallic ferromagnets, as has been shown both experimentally [4] and theoretically [5]. In this respect, $(\mathrm{Ga}, \mathrm{Mn}) \mathrm{As}$, combining semiconducting properties with magnetism, has become a model ferromagnetic semiconductor prospective for developing novel device functionalities.

Recently, we have studied DWs-related phenomena in various ferromagnetic $(\mathrm{Ga}, \mathrm{Mn})$ As nanostructures, composed of either three or four nanowires joined in one point, exploiting the fact that switching the magnetization direction leads to distinct changes in the wire resistance $[6,7]$. In the present paper we report on some new results obtained on four-arm nanostructure fabricated from monocrystalline $\mathrm{Ga}_{0.92} \mathrm{Mn}_{0.08}$ As layer, which exhibits a bistable resistance behavior resulting from switching between two configurations of DWs in the structure.

\section{Experimental}

The p-type $\mathrm{Ga}_{0.92} \mathrm{Mn}_{0.08} \mathrm{As}$ layer, of the thickness of $10 \mathrm{~nm}$, has been grown by low-temperature molecular beam epitaxy (LT-MBE) method on the (001) face of a semi-insulating GaAs substrate. After the growth the layer was annealed in the MBE chamber (in the way described in our previous paper [7]) in order to increase its ferromagnetic transition temperature $T_{\mathrm{C}}$. Magnetic properties of the layer were examined using a superconducting quantum interference device (SQUID) magnetometer revealing $T_{\mathrm{C}}$ of $120 \mathrm{~K}$. Owing to a compressive strain, present in the layer as a result of a lattice mismatch between the layer and the substrate, the layer exhibited in-plane magnetization [8] with easy axes along the in-plane $\langle 100\rangle$ cubic directions and hard axes along two nonequivalent in-plane $\langle 110\rangle$ directions [7].

The four-arm nanostructures consisted of two perpendicularly crossed stripes, of the width of $150 \mathrm{~nm}$ and length of $2 \mu \mathrm{m}$, oriented along various in-plane crystallographic directions, fabricated by means of electron-beam lithography patterning and chemical etching (Fig. 1). The arm terminals were supplied with ohmic contacts, which enabled an electric current flow through any pair of the arms. We measured the longitudinal resistance $R$ between the terminals of opposite arms as a function of in-plane magnetic field $H$ in a range comparable to the magnetic coercivity of the parent $(\mathrm{Ga}, \mathrm{Mn})$ As layer. The measurements were performed using low-frequency $(27 \mathrm{~Hz})$ lock-in technique with a sensing current of $1 \mu \mathrm{A}$. In the following we present the results obtained at the liquid-nitrogen temperature for the nanostructure with the stripes oriented along the two perpendicular $\langle 110\rangle$ directions. 


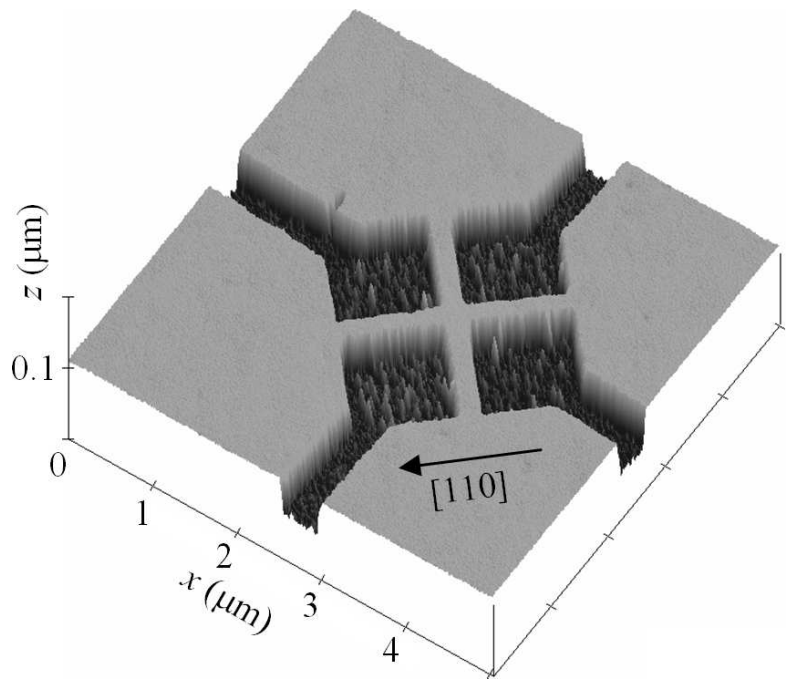

Fig. 1. Atomic force microscope (AFM) image of the four-arm nanostructure.

\section{Results}

As shown in our previous paper [7], while sweeping a magnetic field, parallel to either of the arms, the magnetoresistance (MR) of either of the stripes displays a hysteresis loop, analogous to the magnetization hysteresis loop, with a remnant MR appearing in zero magnetic field. As a consequence, either of the stripes displays a bistable MR behavior, which exhibits two different states in zero magnetic field, depending on the direction of previously applied magnetic field. Moreover, the reversal of the direction of magnetic field results in the exchange of the higher resistance into the lower one in one of the stripes and the opposite exchange in the other stripe, and vice versa.

Figure $2 \mathrm{a}$ shows the MR hysteresis loop obtained at the temperature of $77 \mathrm{~K}$ for the stripe oriented parallel to the $[-110]$ direction under magnetic field applied parallel to the [110] direction. It demonstrates that a pulse of a positive field of 3.5 Oe can switch the zero-field resistance of the stripe from its lower value into the higher one. Consequently, in the next run of the MR hysteresis-loop measurement, performed after a $3 \mathrm{~s}$ pulse of +3.5 Oe magnetic field, the resistance starts from its higher value, as shown in Fig. $2 \mathrm{~b}$. We have attempted to obtain similar effect using an electric current instead of magnetic field. So far, we succeeded in achieving that effect by passing a pulse of dc current of the density of $10^{7} \mathrm{~A} / \mathrm{cm}^{2}$ through the two stripes when they were shorted together. It is illustrated in Fig. 2c where, after the pulse of +3.5 Oe magnetic field, resulting in the higher resistance state of the measured stripe, a $30 \mathrm{~s}$ pulse of dc current was passed through the two stripes. The MR hysteresis loop measured after that (Fig. 2c) started from the lower resistance state, thus demonstrating that the current pulse switched the stripe resistance back to its lower state.

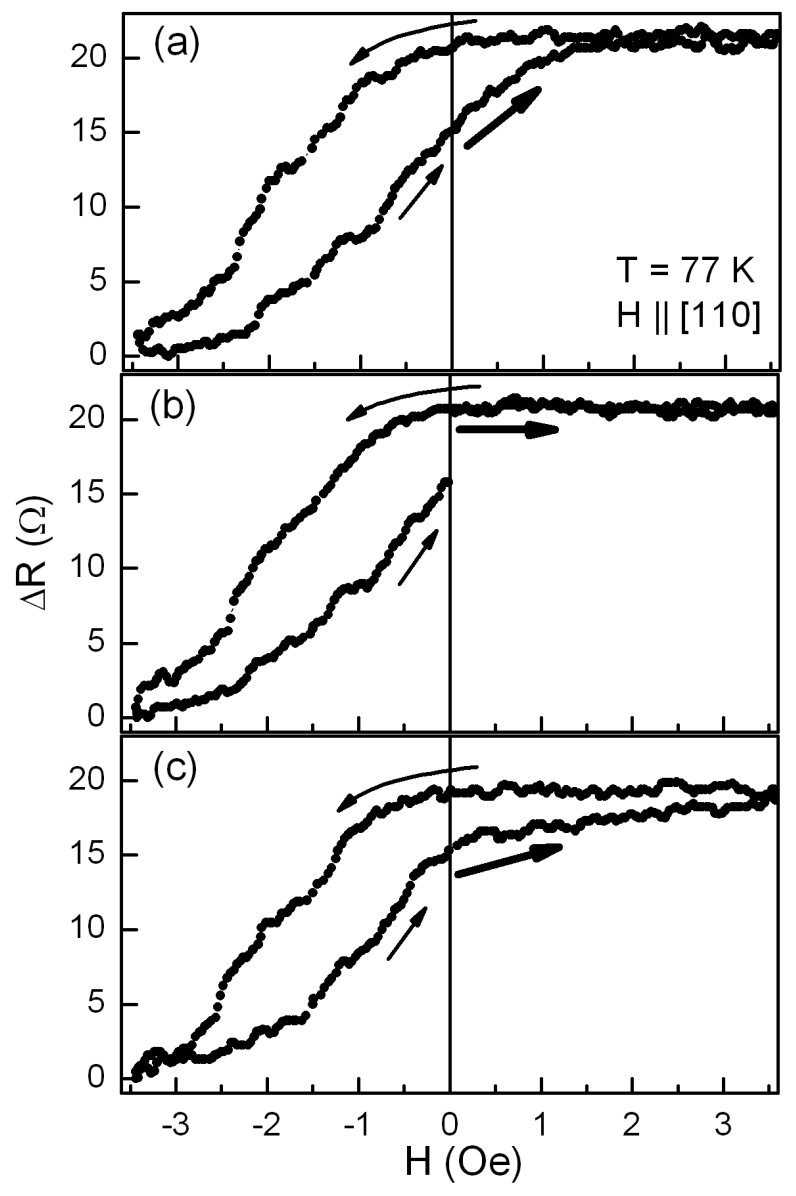

Fig. 2. Three consecutive runs of MR hysteresis loop measurements performed at $77 \mathrm{~K}$ for the stripe oriented along the $[-110]$ crystallographic axis under the in-plane magnetic field swept along the [110] axis. The zero-field resistance of the stripe was about $50 \mathrm{k} \Omega$. Arrows indicate the directions of magnetic field sweep while thick ones denote the initial branches of the loops. (a) Typical MR hysteresis loop with the start and end at the lower zero-field resistance of the stripe. (b) The second run measured after a $3 \mathrm{~s}$ pulse of +3.5 Oe magnetic field. (c) The third run measured after a $3 \mathrm{~s}$ pulse of +3.5 Oe magnetic field followed by a $30 \mathrm{~s}$ pulse of $400 \mu \mathrm{A} \mathrm{dc}$ current passing through the two stripes of the nanostructure.

\section{Discussion and conclusions}

It is crucial for the understanding the bistable MR behavior of the nanostructure that the magnetization vector is always directed along the nanowire axis owing to the patterning-induced magnetic anisotropy [9]. However, this anisotropy does not regard the central region of the nanostructure where the wires join. In this square region a low external magnetic field can switch the local magnetization vector between [100] and [010] easy axes, similarly as it does in the parent layer. This implies the presence of four $45^{\circ} \mathrm{DWs}$, separating the central-part single magnetic domain from the in-arm single domains. Due to limited precision of the lithography process there 
is some small tilt angle between the $\langle 110\rangle$ directions and the stripes' axes, which results in a slight difference between DWs located on the perpendicular stripes, which are no more $45^{\circ} \mathrm{DWs}$. Assuming that the DW resistance increases with the degree of spin misalignment in a wall, all the MR results can be coherently explained by taking into account that switching the magnetization vector between [100] and [010] easy axes in the central domain results in the exchange of the higher-angle DWs into the lower-angle ones in one of the stripes and the opposite exchange in the other stripe [7].

The hysteretic behavior of the nanostructure resistance, which assumes one of its two stable values at zero magnetic field, may be applicable in the memory elements. The four-arm nanostructure represents a four-terminal device that has two complementary outputs, which means that when one pair of arms is in the high-resistance state, the other is in the low-resistance state, and vice versa.

In conclusion, we have designed and investigated four-arm nanostructures having in the scope their possible use in spintronics devices. The nanostructures exhibit a bistable behavior, in which two states of their resistance result from different configurations of domain walls in their central region. By momentary applying a weak magnetic field each of the two stable resistance states can be obtained in zero magnetic field. In addition, we have also demonstrated that a switching between the two resistance states can be also attained by passing a pulse of dc current through the nanostructure.

\section{Acknowledgments}

The authors are grateful to E. Łusakowska for performing the AFM measurements. The work has been supported by the Polish Ministry of Science and Higher Education under grant No. N507 461833.

\section{References}

[1] D.A. Allwood, G. Xiong, C.C. Faulkner, D. Atkinson, D. Petit, R.P. Cowburn, Science 309, 1688 (2005).

[2] S.S.P. Parkin, M. Hayashi, L. Thomas, Science 320, 190 (2008).

[3] G. Tatara, H. Kohno, J. Shibata, Phys. Rep. 468, 213 (2008).

[4] M. Yamanouchi, D. Chiba, F. Matsukura, T. Dietl, H. Ohno, Phys. Rev. Lett. 96, 096601 (2006).

[5] A.K. Nguyen, H.J. Skadsem, A. Brataas, Phys. Rev. Lett. 98, 146602 (2007).

[6] T. Figielski, T. Wosiński, A. Morawski, A. Mąkosa, J. Wróbel, J. Sadowski, Appl. Phys. Lett. 90, 052108 (2007).

[7] T. Andrearczyk, T. Wosiński, T. Figielski, A. Mąkosa, J. Sadowski, Z. Tkaczyk, E. Łusakowska, J. Wróbel, Acta Phys. Pol. A 114, 1049 (2008).

[8] M. Sawicki, K.-Y. Wang, K.W. Edmonds, R.P. Campion, C.R. Staddon, N.R.S. Farley, C.T. Foxon, E. Papis, E. Kamińska, A. Piotrowska, T. Dietl, B.L. Gallagher, Phys. Rev. B 71, 121302(R) (2005).

[9] S. Hümpfner, K. Pappert, J. Wenisch, K. Brunner, C. Gould, G. Schmidt, L.W. Molenkamp, M. Sawicki, T. Dietl, Appl. Phys. Lett. 90, 102102 (2007). 